\title{
Acquiescence in Components Analysis and Multidimensional Scaling of Self-Rating Items
}

\author{
Mark L. Davison and Niyad Srichantra \\ University of Minnesota
}

\begin{abstract}
Earlier work has shown that when multidimensional scaling (MDS) is applied to item intercorrelations, metric MDS implicitly subtracts the standardized person mean (SPM) from responses. As a result, when a metric or nonmetric MDS solution is compared to a components solution, the components solution often contains one component with no counterpart among the scaling dimensions. If self-report items form a ballanced scale and negatively worded items are not reverse scored, the SPM is closely related to several concepts of acquiescence and disacquiescence. In this paper, MDS and components solutions are compared using two balanced self-report item sets. In the Likert self-report attitude item set, the MDS and components solutions were essentially the same. In a set of affective well-being items, the components solution contained a general component with no counterpart among the scaling dimensions. Scores along the general component were substantially correlated with measures of acquiescence and disacquiescence. Results in the second dataser suggest that when the self-report items are balanced and the negatively worded items have not been reverse scored, MDS and components solutions can differ largely with respect to a component closely associated with certain measures of acquiescence. Index terms: acquiescence, attitude measurement, factor analysis, multidimensional scaling, personality measurement, response bias, self-report items.
\end{abstract}

Factor analysis and components analysis remain the most common methods used to analyze the structure of tests or items. More and more, how-

APPLIED PSYCHOLOGICAL MEASUREMENT

Vol. 12, No. 4, December 1988, pp. 339-351

(C) Copyright 1988 Applied Psychological Measurement Inc. 0146-6216/88/040339-13\$1.90 ever, multidimensional scaling (MDS) has been recommended for the same purpose. Schiffman, Reynolds, and Young (1981, p. 15) probably offered the strongest recommendation, emphasizing the experimental, mathematical, and interpretive advantages of MDS. MDS analyses of tests or items have been reported in Farley and Cohen (1974), Guttman (1966), Karni and Levin (1972), Lehmann (1974), Levin, Montag, and Comrey (1983), Napior (1972), Ronen, Kraut, Lingoes, and Aranya (1979), Rounds, Davison, and Dawis (1979), Schlessinger and Guttman (1969), and Silverstein (1987).

Guttman (1966; Schlessinger \& Guttman, 1969) argued that nonmetric MDS provides a more parsimonious representation of test structure. Guttman's research showed a two-dimensional nonmetric MDS in which the major features of a fivefactor solution can be discerned. Lingoes (1971) proved an upper-bound condition that offers some indirect theoretical support for Guttman's argument. It is well known that the correlations among $n$ variables can be reproduced exactly by $n$ components at most. Lingoes (1971) showed that fewer than $n$ dimensions are required to reproduce the order information of a square symmetric matrix in a nonmetric MDs based on a distance model. Hence the upper bound on the number of components needed to reproduce a correlation matrix is greater than the upper bound on the number of dimensions needed to reproduce that same matrix in a nonmetric MDS. 
Relations between MDS and factor analysis have been investigated by Davison (1983, 1985 ), MacCallum (1974), and Tucker (1972). MacCallum presented a comprehensive review covering types of data analyzed, assumptions, objectives, and computational procedures.

Davison (1983, 1985) studied relations between a special case of factor analysis (components analysis) and MDS (both metric and nonmetric) when the two methods were applied to the same intercorrelation matrix. Like MacCallum (1974), Davison (1985) noted that components analysis and metric MDS use closely related computational techniques; both employ theorems introduced into psychology by Eckart and Young (1936) to obtain coordinate estimates, that is, component loadings in components analysis and scale values in metric MDS. In particular, Davison discussed how the MDS dimension scale values compare to the component loadings when both MDS and components analysis are applied to the same intercorrelation matrix. Using an algebraic argument, he showed that metric multidimensional scaling tends to implicitly subtract a source of item variance: the standardized person mean (SPM).

Let $z_{i s}$ be the score of person $s$ on item $i$. For person $s$, the SPM $z_{s}$ is an average score for person $s$ computed across all items: $z_{. s}=(1 / /) \sum_{i} z_{i s}$ where $I$ is the number of items. The SPM represents the overall level of the person's profile of item scores.

Davison (1985) pointed out that the $K$-dimensional metric multidimensional scaling solution contains the first $K$ components of the scalar products matrix $A^{*}$. Let

$z_{i s}^{*}=z_{i s}-z_{. s}$.

A* is an $I \times I$ matrix having rows and columns corresponding to items. The element in row $i$ column $j$ of $\mathbf{B}^{*}$ is $\delta_{i j}^{*}=(\mathbb{1} / S) \sum_{s} z_{i s}^{*} z_{j s}^{*}$, where $S$ is the number of persons. Although the $K$-dimensional metric MDS solution contains the first $K$ components of $A^{*}$, the $K$ components analysis solution contains the first $K$ components of $R_{\text {with elements }}$ $r_{i j}=(1 / S) \Sigma_{s} z_{i s} z_{j s}$. Note that $r_{i j}$ and $\delta_{i j}^{*}$ are identical except that $r_{i j}$ involves standardized scores, $z_{i s}$ and $z_{j s}$, whereas $\delta_{i j}^{*}$ involves standardized scores ex- pressed as deviations about person means, $z_{i s}^{*}$ and $z_{j s}^{*}$.

Because the metric MDS solution contains components of $\mathrm{A}^{*}$, whose elements are sums of crossproducts of the deviation scores, $z_{i s}^{*}=z_{i s}-z_{. s}$, Davison (1985) concluded that metric MDS implicitly subtracts the standardized person mean from the solution. One implication of Davison's (1985) result is the following: Whereas the loadings obtained in a components analysis constitute a coordinate representation reflecting variation in item score profiles due to level, scatter, and shape, the scale values in a metric scaling constitute a coordinate representation reflecting variation due to scatter and shape (but not level).

What is the effect on the solution of subtracting the SPM? In general, it is difficult to characterize the effect. In this article, two special cases are of particular interest. In the first special case, the SPM is zero for everyone (all profiles have the same level), and it can be seen from Equation 1 that subtracting the SPM will have no effect; $A^{*}$ and will be identical, and the metric MDS and components solutions will be the same if the number of dimensions in the MDS solution equals the number of components in the components solution. In practice, of course, the person means are not expected to be exactly equal for all people. However, if there is little variation in person means, then a components solution and a metric MDS solution should be approximately the same if the same numbers of components and dimensions are extracted.

In the second special case, implicitly subtracting the SPM removes a component so that one component in the components solution exists with no counterpart in the metric MDS solution. The "missing" component would have a distinctive form: All loadings along the "missing" component would be equal. In practice, of course, the loadings would not be expected to be exactly equal. Rather, if the data approximate this second special case, then there will be a component along which all loadings are of the same sign and approximately the same magnitude; this component will have no counterpart in a metric MDS solution.

The term "general component" in the remainder of this article refers to any component in which all 
loadings are of the same sign and approximately equal magnitude. Although "general component" has been used to refer to components for which all measures have substantial, nonzero loadings, the definition used here is more restrictive because it requires all loadings to have the same sign and approximately the same magnitude.

Thus, when both metric MDS and components analysis are applied to the same correlation matrix, Davison (1985) pointed out that, because the two methods employ the Eckart-Young decomposition, special cases exist in which a close correspondence between the component loadings and metric MDS scale values is expected.

Furthermore, Davison (1985) showed that these relations between metric MDS and components analysis also tend to hold for nonmetric MDS and components analysis. In his monte carlo studies of simulated correlations, Davison found that metric and nonmetric MDS gave similar solutions when the number of dimensions was held constant. The monte carlo metric MDS and nonmetric MDS solutions tended to stand in the same relationship to the components solution. That is, when Davison simulated special Case 1 (SPM equals zero), the components solution, metric MDS, and nommetric MDS solutions were essentially the same (within a rotation and uniform stretching/shrinking of the MDS axes). When Davison simulated special Case 2 (general component loadings of same magnitude and sign), both the metric and nonmetric MDS SOlutions lacked a general component found in the components solution, but all components other than the general component corresponded to one of the dimensions in the metric MDS solution and one of the dimensions in the nonmetric MDS solution.

In his real data examples, involving subscales of Holland's (1965) Vocational Preference Inventory and the General Aptitude Test Battery (U.S. Department of Labor, 1970), Davison (1985) found that components and nonmetric MDS solutions were very similar to special Case 2 . In a subsequent study, Silverstein (1987) found much the same correspondence between components and nonmetric MDS analyses of subscales from the WAIS-R (WechsIer, 1981), the WISC-R (Wechsler, 1974), and the WPPSI (Wechsler, 1967). In short, when the prox- imity data are correlations, special cases exist in which it can be shown algebraically that certain correspondences exist between components and metric MDS solutions; monte carlo simulations suggest that roughly those same correspondences exist between components and nonmetric MDS solutions; and the correspondence described in the second special case has been observed in components and nonmetric MDS analyses of a number of different instruments.

Focusing on a very specific application, this paper concerns the meaning of the SPM, the variance implicitly removed by metric MDS. The particular application employed here is using MDS to study the intercorrelations of self-report ratings on items that form a balanced scale. A balanced scale is one in which half of the items are positively worded and half are negatively worded. Negatively worded items were not reverse scored, so the "strongly agree" (or "always true of me") response received the highest item score whether or not the item was positively or negatively worded.

In the special case considered here, the SPM is closely associated with the response styles acquiescence and disacquiescence as defined by some authors. If MDS and components analysis yield different coordinate representations of item structure, components analysis will often yield a component with no counterpart among the MDS dimensions, and if certain concepts of acquiescence and disacquiescence are accepted, then this "extra' component will be closely associated with them. Two datasets are used to illustrate the empirical relationship between the SPM and acquiescence/disacquiescence indices. These examples are also used to illustrate two possible relationships between components analysis and nonmetric MDS representations of self-report item structure.

\section{Acquinescence}

Relating acquiescence to MDS and components analysis is complicated by the fact that the literature contains several concepts of acquiescence. One concept holds that acquiescence is itself trait-like in that an individual's tendency to acquiesce is consistent across diverse item sets (Couch \& Ken- 
iston, 1960). This generalized acquiescence concept has largely been discredited (Block, 1965; Rorer, 1965). More recent authors (Ray, 1979, 1983; Ray \& Pratt, 1979; Winkler, Kanouse, \& Ware, 1982, 1984) have conceptualized acquiescence as a response tendency specific to a particular set of items. An item-specific concept of acquiescence implies that any measure of acquiescence must be taken on the set of items to which it refers.

Within the conceptualization of acquiescence as item specific, three statistical definitions of acquiescence have been presented (Chapman \& Bock, 1958; Ray, 1979, 1983; Ray \& Pratt, 1979; Winkler et al., 1982). The definitions differed in the methods used to calculate an acquiescence score for each individual and in their assumptions about the items over which the calculations will be made. All of these authors presume balanced scales, scales containing an equal number of positively and negatively worded items. However, Chapman and Bock (1958) and Winkler et al. (1982), but not Ray (1983), presumed a stronger reversal scale that consists of positively or negatively worded item pairs. For each item pair the negatively worded item is the logical opposite of the positively worded member. Such reversal scales are a special case of balanced scales.

Several algebraic relationships exist between various acquiescence/disacquiescence scores that are important. For convenience, Ray's (1983) score was used as a reference point with which to compare all of the other acquiescence/disacquiescence scores. Ray's acquiescence score is the sum of item responses before reverse scoring negatively worded items: $A_{R}=\sum_{i} x_{i s}$. The more a person agrees with statements, on average, the higher the person's score, $A_{R}$, will be. Because the sum of item responses, $A_{R}$, is simply the elevation in the item responses of a person, Ray equates acquiescence with the elevation of raw scores for a given person.

Chapman and Bock's (1958) acquiescence score equals one-half the difference between the total scores on positively and negatively worded items after reverse scoring negatively worded items. Srichantra (1987; Srichantra \& Davison, 1987) has shown that the Ray and Chapman-Bock scores are linearly related when computed on the same items. If $A_{R}$ is Ray's acquiescence score, $A_{C B}$ is Chapman and Bock's acquiescence score, $I$ is the number of items, and $k$ is the maximum item score, then $A_{C B}=(1 / 2) A_{R}-(I / 4) k$ (Srichantra \& Davison, 1987).

Winkler et al.'s (1982) acquiescence score is the number of logically opposite item pairs for which the person agrees to both members of the pair. If there are $I$ items and $I / 2$ logically opposite item pairs, then the Winkler et al. (1982) acquiescence score can range from 0 to $\mathbb{Z} / 2$. Winkler et al. (1982) also defined a disacquiescence score as the number of logically opposite pairs for which the person disagrees with both members of the pair. Like their acquiescence score, this disacquiescence score can range from 0 to $I / 2$. When the items are dichotomously scored, Srichantra and Davison (1987) have shown that Ray's acquiescence score is linearly related to the difference between Winkler et al.'s acquiescence and disacquiescence scores. That is, if $A_{W}$ and $D_{W}$ represent Winkler's acquiescence and disacquiescence scores, then $A_{R}=A_{W}-D_{W}+I / 2$ (Srichantra \& Davison, 1987). When the items are not dichotomously scored, however, no algebraic relationship exists between Ray's acquiescence score and the acquiescence/disacquiescence scores of Winkler et al. (1982).

Not only is Ray's acquiescence score algebraically related to the acquiescence score of Chapman and Bock (1958) and to the difference between the acquiescence and disacquiescence scores of Winkler et al. (1982) when the items are dichotomously scored, but Ray's acquiescence score is also directly related to the SPM implicitly subtracted by MDS when all item scores have equal variances. Except for multiplication by $\mathbb{1} / I$, the SPIM is a standardized form of Ray's acquiescence score. If negatively worded items have not been reverse scored, then Ray's acquiescence score is a sum of raw item scores whereas the SPM is an average of standardized item scores.

Because Ray's score is linearly related to Chapman and Bock's, the SPM is also linearly related to Chapman and Bock's acquiescence score if computed from standardized item responses (or if all items have equal variances). In addition, because Ray's score is linearly related to the difference between Winkler et al.'s acquiescence and disacquiescence scores when items are dichotomously 
scored, the SPM is linearly related to the difference between Winkler et al.'s (1982) acquiescence and disacquiescence scores if computed from standardized, dichotomous item scores (or if all items have equal variances). In sum, when computed on standardized scores (or when items have equal variances), the SPM is linearly related to $A_{C B}$ and $A_{R}$; and if the items are also dichotomous, the SPM is linearly related to the difference between $A_{W}$ and $D_{W}$.

The two studies described below illustrate the relationship between the SPM and the various acquiescence and disacquiescence scores. Because the Chapman and Bock (1958) and Ray (1979) acquiescence scores are essentially equivalent (linearly related), they were treated as one score, $A_{C B R}$. The examples also illustrate possible relationships between components analysis and MDS coordinate representations of self-report item structure. Study 1 focused on two sets of self-report attitude items forming reversal scales. Study 2 used a set of selfreport affect items forming a balanced, but not a reversal scalle.

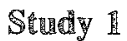

\section{Method}

Participants. The participants were 112 undergraduate students who enrolled in a 10 -week intermediate algebra class at the University of Minnesota. The students came from three classes which were taught by different instructors. All classes were compatible in terms of course content, method of teaching, class activities, and exams.

Attitude measures. The attitude measure covered two related areas: attitudes toward attending the mathematics class and attitudes toward the class in general. Both the Attendance and General scales were reversal scales. The Attendance scale contained 18 items composed of 9 pairs. Within a pair, both items were constructed so that they were as equivalent as possible in content and psychological meaning, but one was worded in the positive direction and the other was worded in the negative direction. The General scale contained 20 items composed of 10 logically opposite pairs. Students responded to items by indicating their level of agreement on a four-point $\mathbb{L i k e r t}$ scale (i.e., strongly agree, agree, disagree, and strongly disagree). The attitude measure was administered to students during the sixth week of the 10 -week quarter.

For each scale, five scores were computed: (1) a total attitude score, (2) the SPM, (3) Winkler et al.'s (1982) acquiescence measure, (4) Winkler ef al.'s disacquiescence measure, and (5) the Chapman-Bock-Ray acquiescence measure. The total attitude score was the sum of the item scores after reverse scoring negatively worded items. The SPM was the average item score for standardized items before reverse scoring negatively worded items. The Winkler et al. acquiescence measure was the number of logically opposite pairs for which the student agreed to both members of the pair. The Winkler et al. disacquiescence score was the number of logically opposite pairs for which the student disagreed with both members of the pair. For the Attendance scale, the Winkler et al. acquiescence and disacquiescence scores could range from 0 to 9, and for the General scale they could range from 0 to 10. The Chapman-Bock-Ray acquiescence score was the sum of item scores before reverse scoring negatively worded items.

\section{盈esulds}

For both artitude scales, Table 1 shows the intercorrelations among the total attitude score, the SPM score, the Winkler acquiescence and disacquiescence scores $\left(A_{W}\right.$ and $\left.D_{W}\right)$, the difference $A_{W}-D_{W}$, and the Chapman-Bock-Ray acquiescence score $\left(A_{C B R}\right)$. On both scales, the correlation between the SPM and the Chapman-Bock-Ray measure of acquiescence was .99 , suggesting that the SPM was virtually identical to their acquiescence measure.

Because $A_{C B R}$ is nearly identical to the SPM and it is linearly related to the difference between the Winkler acquiescence and disacquiescence scores when items are dichotomously scored, it is not surprising to find that the SPM is positively correlated with $A_{W}$ and negatively correlated with $D_{W}$ in both datasets. Although the SPM was significantly correlated with the Winkler et al. measure of acquiescence in both datasets, the correlations of .66 


\begin{tabular}{|c|c|c|c|c|c|c|}
\hline \multirow{2}{*}{ Score } & \multirow{2}{*}{ TA } & \multirow{3}{*}{ SPM } & \multicolumn{4}{|c|}{ Acquiescence Score } \\
\hline & & & & & & \\
\hline TA & & & & & & $w$ w \\
\hline & -- & $-.19 x$ & $-.19 *$ & $-.21 *$ & -.02 & -.13 \\
\hline SPM & .10 & -- & $.99 * x$ & $.66 * x$ & $-.63 * x$ & $.78 * 3 x$ \\
\hline$A_{C B R}$ & .03 & $.99 * *$ & - & $.65 * *$ & $-.63 \times x$ & $.78 \times x$ \\
\hline A & $-.21 x$ & $.50 \times x$ & $.523 x$ & - & $-.28 * *$ & $.83 * x$ \\
\hline$D_{w}^{w}$ & $-.21 \%$ & $-.36 * *$ & $-.36 \pi x$ & .02 & $\cdots$ & $-.77 x * x$ \\
\hline$A_{w}^{w}-D_{w}$ & $18 x^{2}$ & $67 * *$ & $67 * 3 x$ & $.71 * *$ & $-.69 * * x$ & $\cdots$ \\
\hline
\end{tabular}

and .50 for the Attendance and General scales respectively are not nearly so high as those between the SPM and $A_{C B R}$. Nor is the Winkler disacquiescence measure as closely associated with the SPM $(r=-.63$ and $r=-.36)$ as is $A_{C B R}$. The SPM was more highly correlated with the difference between $A_{W}$ and $D_{W}$ in both the Attendance $(r=.78$, $p<.01)$ and the General $(\mathrm{r}=.67, p<.01)$ scales than it was with $A_{W}$ or $D_{W}$ separately. In these data, the SPM implicitly subtracted by metric MDS was virtually perfectly correlated with acquiescence as defined by Chapman and Bock (1958) and Ray (1983), and it was substantially correlated with acquiescence, disacquiescence, and the difference between acquiescence and disacquiescence as defined by Winkler et al. (1982).

For this particular dataset, components analysis and the nonmetric scaling program KYST (Kruskal, Young, \& Seery, 1973) gave very similar results when applied to the inter-item correlations before reverse scoring. Components solutions were obtained in one to five components/dimensions. For Components 1 through 5 respectively, the eigenvalues were $4.41,2.09,1.85,1.48$, and 1.17 in the solutions based on the Attendance scale and $6.54,2.26,1.68,1.34$, and 1.17 in the solutions based on the General scale. All components beyond the fifth had eigenvalues less than 1. For the onethrough five-dimensional KYST solutions, stress statistics were $.29, .14, .09, .06$, and .04 in solutions based on the Attendance scale and $.09, .08$,
$.05, .04$, and .03 for solutions based on the General scale.

The first five components did not contain a general component along which all items had loadings of the same sign. This result suggests that these data correspond to the first case described above in which components analysis and nonmetric scaling give essentially the same coordinate representations. Congruence coefficients were computed for solutions with equal numbers of components/dimensions. Both the component and MDS solutions were placed in a principal axis orientation. Where necessary, dimensions were reflected. For solutions based on the Attendance scale items with one to five components/dimensions respectively, the congruence coefficients were $.98, .95, .96, .95$, and .95 . Using the General scale items, the congruence coefficients were $.99, .91, .91, .93$, and .92.

The component by dimension congruence coefficients were also computed and are shown in Table 2. Congruence coefficients for matching components/dimensions are shown along the diagonals in each of the submatrices. Although there are exceptions, most of the coefficients are greater than 90. The lowest values occur for Components/Dimensions 4 and 5 in the five component/dimension solutions using the General scale. Some of the mismatch can be attributed to incongruent rotations, because the fourth component (dimension) matches the fifth dimension (component) almost as well as 
the fourth component matches the fourth dimension and almost as well as the fifth component matches the fifth dimension.

Table 3 displays the correspondence between the four-component and four-dimensional solutions for the Attendance scale. To arrive at the scale values in Table 3, the signs of all items were reflected (where necessary) along a dimension to bring them into correspondence with the signs of loadings, and the scale values were normalized so their total sum of squares (across all four dimensions) equaled the sum of squared component loadings. This transformation of the nonmetric scaling solution is consistent with the fact that the MDS solution is determined only up to a rotation, translation of the origin, and uniform stretching or shrinking of the axes. With a few exceptions, the component loading for each item roughly equals the corresponding nonmetric scale value in sign and magnitude.

Even though components and nonmetric MDS solutions in equal numbers of components/dimensions are very similar, it should be noted that two researchers, one of whom uses components analysis and one of whom uses nonmetric MDs, might not arrive at the same coordinate representation. Because nonmetric MDS can often reproduce data well in a small number of dimensions and because a tradition exists in MDS of retaining low dimensionality solutions (particularly two-dimensional solutions), the researcher using MDS may well retain a solution with fewer dimensions than that retained by the researcher using components analysis.

Thus, these data show that the SPM was virtually identical to the Chapman-Bock-Ray acquiescence score. Although the correlations were not near $\mathbb{1}$ in absolute value, the SPM was substantially correlated with Winkler's acquiescence score, disacquiescence score, and the difference between the two. The difference $A_{W}-D_{W}$ was more highly correlated with the SPM than was either $A_{W}$ or $D_{W}$ separately. In these data, the relationship between the MDS and components solutions in up to five components/dimensions approximated Davison's

Table 2

Component by Dimension Congruence Coefficients for the Attendance and General Scales

\begin{tabular}{|c|c|c|c|c|c|c|c|c|c|c|}
\hline \multirow{2}{*}{$\begin{array}{c}\text { Components } \\
\text { and Dimensions }\end{array}$} & \multicolumn{5}{|c|}{ Attendance Dimension } & \multicolumn{5}{|c|}{ General Dimension } \\
\hline & 1 & 2 & 3 & 4 & 5 & 1 & 2 & 3 & 4 & 5 \\
\hline \multicolumn{11}{|l|}{ One } \\
\hline 1 & .979 & & & & & .989 & & & & \\
\hline \multicolumn{11}{|l|}{ Two } \\
\hline 1 & .976 & .045 & & & & .993 & .042 & & & \\
\hline 2 & .056 & .899 & & & & .004 & .679 & & & \\
\hline \multicolumn{11}{|l|}{ Three } \\
\hline 1 & .986 & .044 & .022 & & & .994 & .035 & .007 & & \\
\hline 2 & .031 & .949 & .119 & & & .000 & .770 & .380 & & \\
\hline 3 & .013 & .127 & .940 & & & .000 & .260 & .849 & & \\
\hline \multicolumn{11}{|l|}{ Four } \\
\hline 1 & .990 & .023 & .007 & .011 & & .996 & .025 & .008 & .045 & \\
\hline 2 & .013 & .970 & .043 & .033 & & .003 & .902 & .298 & .253 & \\
\hline 3 & .006 & .044 & .981 & .087 & & .001 & .236 & .910 & .243 & \\
\hline 4 & .040 & .106 & .033 & .773 & & .025 & .215 & .132 & .665 & \\
\hline \multicolumn{11}{|l|}{ Five } \\
\hline 1. & .993 & .017 & .019 & .022 & .010 & .997 & .017 & .002 & .044 & .039 \\
\hline 2 & .014 & .978 & .096 & .083 & .038 & .004 & .964 & .212 & .109 & .015 \\
\hline 3 & .017 & .116 & .962 & .213 & .026 & .005 & .188 & .944 & .177 & .057 \\
\hline 4 & .034 & .064 & .184 & .833 & .380 & .020 & .082 & .054 & .697 & .615 \\
\hline 5 & .024 & .048 & .049 & .035 & .888 & .007 & .085 & .149 & .610 & .662 \\
\hline
\end{tabular}

Downloaded from the Digital Conservancy at the University of Minnesota, http://purl.umn.edu/93227. May be reproduced with no cost by students and faculty for academic use. Non-academic reproduction requires payment of royalties through the Copyright Clearance Center, http://www.copyright.com/ 
Table 3

Components (Con) and Dimensions (Dim) from the

Unrotated Four Components Solution and Four-Dimensional Nonmetric MDS Solution for the Attendance Scale (Coefficient of Congruence $=.95$ )

\begin{tabular}{|c|c|c|c|c|c|c|c|c|}
\hline Item & $\begin{array}{c}\text { Com } \\
1\end{array}$ & $\begin{array}{c}\text { Dim } \\
1\end{array}$ & $\begin{array}{c}\text { Com } \\
2\end{array}$ & $\begin{array}{c}\text { Dim } \\
2\end{array}$ & $\begin{array}{c}\text { Com } \\
3\end{array}$ & $\begin{array}{c}\text { Dim } \\
3\end{array}$ & $\begin{array}{c}\text { Com } \\
4\end{array}$ & $\begin{array}{c}\text { Dim } \\
4\end{array}$ \\
\hline 1 & .485 & .536 & .425 & .386 & -.030 & -.015 & .443 & .329 \\
\hline 2 & .262 & .314 & -.402 & -.433 & -.361 & -.358 & .213 & .239 \\
\hline 3 & .638 & .743 & .149 & .180 & -.285 & -.280 & .011 & .159 \\
\hline 4 & .256 & .384 & -.467 & -.453 & .280 & .268 & .440 & .324 \\
\hline 5 & .718 & .762 & .343 & .211 & .102 & .072 & .312 & .068 \\
\hline 6 & .355 & .409 & .329 & .436 & .414 & .355 & -.530 & -.398 \\
\hline 7 & .136 & .200 & .216 & .327 & -.551 & -.592 & -.335 & -.309 \\
\hline 8 & .469 & .551 & -.390 & -.247 & .505 & .476 & .153 & .042 \\
\hline 9 & .390 & .636 & -.095 & -.270 & -.034 & -.137 & .112 & -.417 \\
\hline 10 & -.566 & -.617 & .064 & .048 & -.027 & -.077 & -.015 & -.336 \\
\hline 11 & -.477 & -.468 & .315 & .308 & .475 & .349 & -.050 & -.160 \\
\hline 12 & -.691 & -.684 & -.044 & -.115 & .113 & .127 & .162 & .115 \\
\hline 13 & -.145 & -.126 & -.689 & -.678 & .106 & .168 & -.324 & -.318 \\
\hline 14 & -.585 & -.567 & -.236 & -.271 & -.274 & -.320 & -.183 & -.081 \\
\hline 15 & -.534 & -.525 & -.240 & -.239 & -.352 & -.264 & .488 & .249 \\
\hline 16 & -.502 & -.479 & .260 & .221 & .484 & .421 & .217 & .199 \\
\hline 17 & -.469 & -.437 & .545 & .423 & -.331 & -.233 & .207 & .231 \\
\hline 18 & -.673 & -.633 & .107 & .168 & .157 & .042 & .155 & .064 \\
\hline
\end{tabular}

(1985) first special case. When his first case holds exactly, the SPM is equal for every person, and the components and metric MDS solutions will be identical. When analyzed in up to five components, the components and nonmetric scaling representations of these attitude items were highly similar.

\section{Study 2}

\section{Method}

Participants. The participants were 638 residents drawn from 30 nursing homes in the mountain states region. Because of missing data, sample sizes in the various analyses ranged from 621 to 638.

Affect measure. Trained interviewers orally administered a questionnaire to each resident in the study. The questionnaire asked about physical, psychological, and social characteristics of the resident. Twelve affective well-being items were included, seven negative-affect items and five pos- itive-affect items. The present analysis focused on these affect items.

Negative-affect items asked about the frequency with which the resident felt bothered by nerves, sad, depressed, worried, tense, suicidal, and lonely. The positive-affect items asked how often the resident felt happy, interested in life, fresh and rested in the morning, had something to look forward to, and cheerful. Because the various definitions of acquiescence assume a balanced scale, two negatively worded items were eliminated (suicide and worry). Residents responded on a four-point scale ranging from "Most of the time" to "None of the time" with "I don't know" as an additional response alternative. Interviewers administered the items orally and recorded the response (or "No response" if the resident did not reply).

Three scores were derived from responses to the affective well-being items. The first was an Acquiescence score, the sum of raw item responses before reverse scoring negative-affect items, which is Ray's $(1979,1983)$ acquiescence score. While 
the scoring formula is essentially equivalent (linearly related) to that of Chapman and Bock (1958), because the items do not constitute a reversal scale the acquiescence score is technically not that of Chapman and Bock. Because the items did not constitute a reversal scale, the Winkler et al. (1982) acquiescence score could not be computed on these data.

The second score was the SPM, the sum of standardized item scores before reverse scoring negative-affect items. The third score was the Affective Well-Being score, the sum of raw item scores after reverse scoring negative-affect items.

\section{瑅esu謟S}

The correlation between the acquiescence score and the SPM was 1. For purposes of correlational analyses, these two measures were treated as one. As in the previous study, acquiescence as computed by Ray was virtually identical to the SPM implicitly subtracted from responses by metric MDS.

For this particular dataset, the unrotated components solution contained a general component, Component 2, with no counterpart among the nonmetric scaling dimensions. Nonmetric scaling and components analyses in one through four dimensions/components were performed on the intercorrelations of items before reverse scoring the neg- ative-affect items. For Components 1 through 4, the eigenvalues were $4.08,1.44, .88$, and .69 . Stress statistics for the one- through four-dimensional KYST solutions all equaled .01 .

To further illustrate the possible relationships between the components and nonmetric MDS solutions, Table 4 contains the loadings from the unrotated four-component solution and the scale values from the three-dimensional nonmetric scaling solution. Where necessary, dimension scale values in Table 4 were reflected. The sum of squared scale values was normalized so as to equal the sum of squared factor loadings along Components 1,3 , and 4.

Component 2 in Table 4 is the general component along which all items have loadings of the same sign and approximately the same magnitude. This second component is closely associated with the SPM and Ray's acquiescence score. The correlation of Component 2 scores with SPM scores was 99 . The correlation between Component 2 scores and Ray's acquiescence score was also 99. Component 2 has no counterpart among the scaling dimensions.

Other than Component 2, all three remaining components closely match a corresponding dimension. Scale values along Dimension 1 closely match Component 1 loadings in sign and magnitude. Dimension 2 matches Component 3 and Dimension

Table 4

Components (Com) and Dimensions (Dim) from the Unrotated Four Components Solution and

Three-Dimensional Nonmetric MDS Solution for the Affective Well-Being Scale (Coefficient of Congruence $=.97$ )

\begin{tabular}{rccccrrr}
\hline \hline Item & Com & Dim & Com & Com & Dim & Com & Dim \\
\hline 1 & -.742 & -.811 & .247 & -.117 & -.113 & .328 & .143 \\
2 & -.610 & -.713 & .477 & .036 & -.074 & .042 & -.090 \\
3 & -.545 & -.635 & .351 & .491 & .415 & .106 & .083 \\
4 & -.495 & -.587 & .501 & -.142 & -.070 & -.637 & -.362 \\
5 & -.701 & -.756 & .352 & -.128 & -.103 & .241 & .170 \\
6 & .544 & .618 & .330 & -.574 & -.394 & .238 & .092 \\
7 & .675 & .723 & .351 & .251 & .125 & -.052 & -.125 \\
8 & .747 & .768 & .356 & .159 & .095 & -.024 & -.076 \\
9 & .652 & .705 & .446 & -.228 & -.108 & -.037 & .031 \\
10 & .623 & .688 & .298 & .344 & .226 & .204 & .131 \\
\hline
\end{tabular}

Downloaded from the Digital Conservancy at the University of Minnesota, http://purl.umn.edu/93227. May be reproduced with no cost by students and faculty for academic use. Non-academic reproduction requires payment of royalties through the Copyright Clearance Center, http://www.copyright.com/ 
3 matches Component 4. For the three-dimensional nonmetric scaling dimensions and the three components other than Component 2, the congruence coefficient was 97.

Similarly close matches occurred for other combinations of dimensions and components. For the one-dimensional scaling solution and two-component solution, the congruence coefficient (deleting Component 2) was .99. For the two-dimensional scaling solution and three-component solution, the congruence coefficient (after deleting Component 2) was 94.

Component by dimension congruence coeffcients were also computed and are shown in Table 5. Congruence coefficients for matching components/dimensions are shown on the diagonals of Table 5. The congruence coefficients in Table 5 confirm that the general component has no counterpart in the two- or three-dimensional solutions. The largest congruence coefficient for the general component and a dimension is .149. All other components have congruence coefficients of greater than .90 with a corresponding dimension.

As in Study 1, the SPM in Study 2 was virtually identical to Ray's acquiescence score. However, the relationship between the components solution and the nonmetric-scaling solution was quite unlike that in Study 1. In Davison's (1985) special Case 2 , a component exists in the components solution with no counterpart among the MDS dimensions. That "extra" component has a very distinctive form: All items have approximately equal loadings. In the present study, Component 2 approximated this distinctive form, and it had no counterpart among the nonmetric scaling dimensions. Component scores along the "extra" component correlated almost perfectly with the SPM (.99) and with Ray's acquiescence measure (.99). The component with no counterpart among the nonmetric MDS dimensions was closely linked to acquiescence as defined by Ray (1979). The data in Study 2 lead to another important point: The general component need not be the first component. Unless the variance associated with elevation (SPM) is substantial, the general component associated with elevation will not be the one accounting for the most variance. In Study 2, the general component associated with the SPM emerged as the second component, not the first.

\section{Discission}

Does MDS implicitly correct for acquiescence? Metric MDS implicitly subtracts the standardized person mean. Whether a researcher considers this a correction for acquiescence will depend on how the items are constructed, how the items are scored, and how the researcher defines acquiescence. When

Table 5

Component by Dimension Congruence Coefficients for the Well-Being Scale

\begin{tabular}{|c|c|c|c|}
\hline \multirow[b]{2}{*}{ Component } & \multicolumn{3}{|c|}{ Dimension } \\
\hline & 1 & 2 & 3 \\
\hline \multicolumn{4}{|c|}{ One Matching Component and Dimension } \\
\hline Component 1 & .994 & & \\
\hline General (Component 2) & .037 & & \\
\hline \multicolumn{4}{|c|}{ Two Matching Components and Dimensions } \\
\hline Component 1 & .995 & .015 & \\
\hline Component 3 & .056 & .903 & \\
\hline General (Component 2) & .038 & .080 & \\
\hline \multicolumn{4}{|c|}{ Three Matching Components and Dimensions } \\
\hline Component 1 & .999 & .016 & .026 \\
\hline Component 3 & .018 & .974 & .017 \\
\hline Component 4 & .018 & .052 & .927 \\
\hline General (Component 2) & .027 & .027 & .149 \\
\hline
\end{tabular}

Downloaded from the Digital Conservancy at the University of Minnesota, http://purl.umn.edu/93227. May be reproduced with no cost by students and faculty for academic use. Non-academic reproduction requires payment of royalties through the Copyright Clearance Center, http://www.copyright.com/ 
the items form a balanced scale and negatively worded items are not reverse scored, the SPM implicitly subtracted by MDS is linked to several measures of acquiescence. Combining the theoretical and empirical results indicates that the SPM subtracted by metric MDS seems most closely linked to acquiescence as defined by Ray (1979) and, if the items also form a reversal scale, to acquiescence as defined by Chapman and Bock (1958). Under these conditions, the SPM is a standardized form of the Ray or Chapman-Bock acquiescence measures. For the attitude datasets, in which the Ray and Chapman-Bock acquiescence scores were essentially identical (linearly related), the correlations of the SPM and the Chapman-Bock-Ray acquiescence measures were .99 and 1 . In the affective well-being data, the correlation between the SPM and Ray's acquiescence score was 1 to two decimal places.

The SPM seems less closely linked to acquiescence and disacquiescence as defined by Winkler et al. (1982) than with acquiescence as defined by Chapman and Bock (1958) or Ray (1979). Both the theoretical and empirical results suggest that the SPM is more highly associated with the difference between Winkler et al.'s (1982) acquiescence and disacquiescence scores than with either Winkler's acquiescence or disacquiescence scores considered separately.

Although the SPM may be nearly identical to acquiescence as defined by Chapman and Bock (1958) and Ray (1979) when the items form a balanced (or reversal) scale and negatively worded items have not been reverse scored, it seems unlikely that the SPM is pure response-style variance. Srichantra and Davison (1987) reported 18 correlations ( 9 for the Attendance and 9 for the General scale) between the Chapman-Bock-Ray acquiescence measure (which was virtually identical to the SPM) and several class attendance and class achievement variables. Three of the 18 correlations were significant at the .05 level. In no instance did the SPM account for as much as $10 \%$ of the variance in any of the class attendance or achievement variables. Because the SPM was virtually identical to the Chapman-Bock-Ray acquiescence score, it must be concluded that the SPM carries a portion of valid, trait-related variance, but apparently a very small portion.

If MDS corrects for acquiescence by implicitly subtracting the SPM, Study 1 suggests that sometimes the "correction" has no effect. In that study, components and nonmetric MDS solutions in up to five dimensions were nearly identical. On the other hand, Study 2 illustrates how components solutions and nonmetric scaling solutions can differ in that the components solution contains a component with no counterpart among the scaling dimensions. In solutions with up to four dimensions and five components, only Component 2 lacked a counterpart among the nonmetric scaling dimensions. Component 2 scores correlated almost perfectly with Ray's acquiescence scores and SPM scores. Nonmetric MDS "corrected" for acquiescence as defined by $\mathbb{R}$ ay in that it included no dimension corresponding to the component whose component scores correlated almost perfectly with Ray's acquiescence scores.

In applying the results of this paper, note that MDS seems to implicitly remove the elevation (SPM) variance of scores, and if a component exists that corresponds to the elevation variance, that component will not be represented by a dimension in the MDS solution. MDS will eliminate acquiescence variance from the representation of item structure only if the elevation variance corresponds to acquiescence variance. At a minimum, for the SPM to represent acquiescence variance, the items must be balanced and the items must not be reverse scored. Only when items are not reverse scored will differences in the tendency to agree be reflected by differences in the elevation (SPM) of item responses. True balancing of items requires that the items be half favorably (positively) and half unfavorably (negatively) worded. It also requires that acquiescence contribute an equal proportion of variance to the positively and negatively worded items.

In short, if MDS is to eliminate acquiescence variance from the representation of structure, the researcher must consciously design the items and score the items so that elevation (SPM) variance will correspond to acquiescence variance. When the items are not so appropriately designed and scored, the elevation variance will not correspond 
to acquiescence; MDS may still remove the general component, if any, but the component removed will now correspond to something other than acquiescence.

One last issue deserves mention. The acquiescence measures discussed here (Chapman \& Bock, 1958; Ray, 1983; Winkler et al., 1982) come from the attitude literature. Jackson and his colleagues (Morf \& Jackson, 1972; Rogers, 1971; Voyce \& Jackson, 1977) have convincingly argued that two acquiescence processes exist: the tendency to agree to statements and the tendency to ascribe characteristics to oneself. According to these authors, the former tendency is more pronounced in attitude responses while the latter is more pronounced in personality responses. However, the vast majority of personality questionnaires appear to confound the two tendencies so that the variance associated with the tendency to agree is virtually identical to the variance associated with the tendency to ascribe characteristics to oneself. Therefore, for purposes of the arguments in this paper, similar reasoning applies to elevation of responses in personality and attitude items. Because acquiescence does correspond to a somewhat different process in the two domains, however, it is possible that the statistical property, elevation of responses, is more closely tied to the psychological phenomenon, acquiescence, in one domain than in the other.

\section{Referentecs}

Block, I. (1965). The challenge of response sets. New York: Appleton-Century-Crofts.

Chapman, L. J., \& Bock, D. R. (1958). Components of variance due to acquiescence and content in the $F$ Scale measure of authoritarianism. Psychological Bulletin, 55, 328-333.

Couch, A., \& Keniston, K. (1960). Yeasayers and naysayers: Agreeing response set as a personality variable. Journal of Abnormal and Social Psychology, 60 , $151-174$

Davison, M. L. (1983). Mullidimensional scaling. New York: Wiley.

Davison, M. L. (1985). Multidimensional scaling versus components analysis of test intercorrelations. Psychological Bulletin, 97, 94-105.

Eckart, C., \& Young, G. (1936). The approximation of one matrix by another of lower rank. Psychometrika, 1, 211-218.
Farley, F. H., \& Cohen, A. (1974). Common-item effects and the smallest space analysis of structure. Journal of Applied Psychology, 81, 766-822.

Guttman, L. (1966). Order analysis of correlation matrices. In R. B. Cattell (Ed.), Handbook of multivariate experimental psychology (pp. 439-458). Chicago: Rand McNally.

Holland, J. L. (1965). Manual for the vocational preference inventory (6th ed.). Palo Alto CA: Consulting Psychologists Press.

Karni, E. S., \& Levin, J. (1972). The use of smallest space analysis in studying scale structure: An application to the California Psychological Inventory. Jour nal of Applied Psychology, 56, 341-346.

Kruskal, J. B, Young, F. W., \& Seery, J. B. (1973). How to use KYST: A very flexible program to do multidimensional scaling and unfolding. Unpublished manuscript, Bell Laboratories.

Lehmann, D. R. (1974). Some alternatives to linear factor analysis for variable grouping applied to buyer behavior variables. Journal of Marketing Research, $11,206-213$.

Levin, J., Montag, I., \& Comrey, A. I. (1983). Comparison of multitrait-multimethod factor and smallest space analysis on personality scale data. Psychological Reports, 53, 591-596.

Lingoes, J. C. (1971). Some boundary conditions for a monotone analysis of symmetric matrices. Psychometrika, 36, 195-203.

MacCallum, R. C. (1974). Relations between factor analysis and multidimensional scaling. Psychological Bulletin, 81, 505-516.

Morf, M. E., \& Jackson, D. N. (1972). An analysis of two response styles: True responding and item endorsement. Educational and Psychological Measurement, 32, 329-353.

Napior, D. (1972). Nonmetric multidimensional techniques for summated ratings. In R. N. Shepard, A. K. Romney, and S. B. Nerlove (Eds.), Multidimensional scaling: Theory and applications in the behavioral sciences (Vol. 1, pp. 157-178). New York: Seminar Press.

Ray, J. J. (1979). Is the acquiescent response style not so mythical after all? Some results from a successful balanced $F$ scale. Journal of Personality Assessment, $43,638-643$.

Ray, J. J. (1983). Reviving the problem of acquiescent response bias. Journal of Social Psychology, 121, 8196.

Ray, J. I., \& Pratt, G. I. (1979). Is the influence of acquiescence on "catchphrase" type attitude scale items not so mythical after all? Australian Journal of Psychology, 31, 73-78.

Rogers, T. B. (1971). The process of responding to personality items: Some issues, a theory, and some research. Multivariate Behavioral Research Monographs, 6(2). 
Ronen, S., Kraut, A. I., Lingoes, J. C., \& Aranya, N. (1979). A nonmetric scaling approach to taxonomies of employee work motivation. Multivariate Behavioral Research, 14, 378-401.

Rorer, L. G. (1965). The great response style myth. Psychological Bulletin, 63, 129-156.

Rounds, J. B., Jr., Davison, M. L., \& Dawis, R. V. (1979). The fit between Strong-Campbell interest inventory general occupational themes and Holland's hexagonal model. Journal of Vocational Behavior, 15 , 303-315.

Schiffman, S. S., Reynolds, M. L., \& Young, F. W. (1981). Introduction to multidimensional scaling. New York: Academic Press.

Schlessinger, I. M., \& Guttman, L. (1969). Smallest space analysis of intelligence and achievement tests. Psychological Bulletin, 71, 95-100.

Silverstein, A. B. (1987). Multidimensional scaling vs. factor analysis of Wechsler's intelligence scales. Journal of Clinical Psychology, 43, 381-386.

Srichantra, N. (1987). Multidimensional scaling: Does it remove acquiescence from the representation of Likert attitude item structure. Unpublished doctoral dissertation, University of Minnesota, Minneapolis.

Srichantra, N., \& Davison, M. L. (1987). On measures of acquiescence and disacquiescence in Likert selfrating items. Unpublished manuscript, University of Minnesota, Minneapolis.

Tucker, L. R. (1972). Relations between multidimensional scaling and three-mode factor analysis. Psychometrika, 37, 3-27.

U.S. Department of Labor (1970). Manual for the USTES. Washington DC: U.S. Government Printing Office.

Voyce, C. D., \& Jackson, D. N. (1977). An evaluation of a threshold theory for personality assessment. $E d$ - ucational and Psychological Measurement, 37, 383408.

Wechsler, D. (1967). Manual for the Wechsler Preschool and Primary Scale of Intelligence. New York: Psychological Corporation.

Wechsler, D. (1974). Manual for the Wechsler Intelligence Scale for Children-Revised. New York: Psychological Corporation.

Wechsler, D. (1981). WAIS-R Manual: Wechsler Adult Intelligence Scale-Revised. New York: Psychological Corporation.

Winkler, J. D., Kanouse, D. E., \& Ware, I. E., Ir. (1982). Controlling for acquiescence response set in scale development. Journal of Applied Psychology, $67,555-561$.

Winkler, J. D., Kanouse, D. E., \& Ware, J.E., Ir. (1984). Does acquiescence distort attitude scale structure: Round and round with Ray. Journal of Applied Psychology, 69, 356-358.

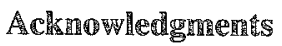

Funding for data collection in Study 2 was provided by the W.K.Kellogg Foundation, the Robert Wood Johnson Foundation, and the Health Care Financing Administration.

\section{Aldithor's Address}

Send requests for reprints or further information to Mark $\mathbb{L}$. Davison, Department of Educational Psychology, University of Minnesota, 178 Pillsbury Dr. S.E., Minneapolis MN 55455, U.S.A. 